\title{
Reliability of contour-based volume calculation for radiosurgery
}

\section{Laboratory investigation}

\author{
Lijun Ma, Ph.D., ${ }^{1}$ Arjun Sahgal, M.D., ${ }^{4}$ Ke Nie, Ph.D., ${ }^{1}$ Andrew Hwang, Ph.D., ${ }^{1}$ \\ Aliaksandr Karotki, Ph.D., ${ }^{4}$ Brian Wang, Ph.D., ${ }^{3}$ Dennis C. Shrieve, M.D., Ph.D., ${ }^{3}$ \\ Penny K. Sneed, M.D., ${ }^{1}$ Michael McDermott, M.D., ${ }^{1}$ and David A. Larson, M.D., Ph.D. ${ }^{1,2}$ \\ ${ }^{I}$ Department of Radiation Oncology, University of California, San Francisco; ${ }^{2}$ Washington \\ Fremont Hospital Gamma Knife Center, Fremont, California; ${ }^{3}$ Department of Radiation Oncology, \\ University of Utah, Salt Lake City, Utah; and ${ }^{4}$ Department of Radiation Oncology, Sunnybrook Odette \\ Cancer Centre, Princess Margaret Hospital, University of Toronto, Ontario, Canada
}

\begin{abstract}
Object. Determining accurate target volume is critical for both prescribing and evaluating stereotactic radiosurgery (SRS) treatments. The aim of this study was to determine the reliability of contour-based volume calculations made by current major SRS platforms.

Methods. Spheres ranging in diameter from 6.4 to $38.2 \mathrm{~mm}$ were scanned and then delineated on imaging studies. Contour data sets were subsequently exported to 6 SRS treatment-planning platforms for volume calculations and comparisons. This procedure was repeated for the case of a patient with 12 metastatic lesions distributed throughout the brain. Both the phantom and patient datasets were exported to a stand-alone workstation for an independent volume-calculation analysis using a series of 10 algorithms that included approaches such as slice stacking, surface meshing, point-cloud filling, and so forth.

Results. Contour data-rendered volumes exhibited large variations across the current SRS platforms investigated for both the phantom $(-3.6 \%$ to $22 \%)$ and patient case $(1.0 \%-10.2 \%)$. The majority of the clinical SRS systems and algorithms overestimated the volumes of the spheres, compared with their known physical volumes. An independent algorithm analysis found a similar trend in variability, and large variations were typically associated with small objects whose volumes were $<0.4 \mathrm{~cm}^{3}$ and with those objects located near the end-slice of the scan limits.

Conclusions. Significant variations in volume calculation were observed based on data obtained from the SRS systems that were investigated. This observation highlights the need for strict quality assurance and benchmarking efforts when commissioning SRS systems for clinical use and, moreover, when conducting multiinstitutional crossSRS platform clinical studies.

(http://thejns.org/doi/abs/10.3171/2012.7.GKS121016)
\end{abstract}

\section{KEY WORDS - volume calculation - volume reconstruction • stereotactic radiosurgery $\quad \bullet \quad$ treatment planning}

$\mathrm{B}$ EFORE an SRS procedure commences, accurate determination of the target volume is critical to ensure the suitability of performing SRS and the dose selection. During SRS treatment planning, volumes of contoured structures of interest are important in evaluating treatment plan quality and commonly comprise various treatment planning criteria such as Paddick indices, ${ }^{18,19}$ mean dose and integral dose, and various dose-volume histogram criteria (for example, the 12-Gy volume). After SRS, changes in target volume are the

Abbreviations used in this paper: DICOM-RT = Digital Imaging and Communication in Medicine-Radiotherapy; FEM = finite element method; SRS = stereotactic radiosurgery. means by which treatment responses are judged, $, 23,25$ and segmented volume-based thresholds have recently been introduced. ${ }^{8}$

The most common method used to obtain the volume of a structure is based on user-defined planar contours, mostly found on serial imaging studies such as CT or MR imaging studies. Technically, all contour lines are digitally recorded as sets of vector endpoints originating from the imaging center during the delineation process. The data are then stored under an image-based or stereotactic framed-based coordinate system. These vector endpoints are also embedded in the current DICOM-RT protocol for the structure data exchange. Currently, there is no standardized method for constructing a volume in 3 dimensions from such a data set. Furthermore, current 
SRS treatment platforms use a variety of methods for this task, resulting in noticeable variability in target volume despite the same contour data set. This phenomenon has been noted in our previous study. ${ }^{15}$

The goal of this study was 2-fold: 1) to investigate the extent of such variability across current SRS platforms and to benchmark the accuracy of the volume calculation process among these systems; and 2) to investigate potential pitfalls that cause such variability and investigate solutions to the problem. For this purpose, this study was carried out using phantoms of known object sizes as well as an analysis of a patient case.

\section{Methods}

We investigated the following platforms in this study: 1) Leksell GammaPlan ${ }^{11,12}$ (Elekta AB); 2) MultiPlan ${ }^{1}$ (Accuray); 3) iPlan 2,10,21 (Brainlab AG); 4) TomoPlan ${ }^{16,17}$ (TomoTherapy); 5) Pinnacle ${ }^{4,5}$ (Philips Medical Systems); and 6) MIM $5^{26}$ (MIM Software). Note that third-party systems, such as Pinnacle and MIM5, do not manufacture dedicated hardware for SRS treatments, but they do support across-platform SRS treatment plan imports and evaluations; therefore, they were included in this study for the purpose of comparison.

The first step of this study was based on a phantom, which was designed using a series of acrylic spheres with precisely machined dimensions (TAP Plastics). The diameters of the spheres were further verified using a digital caliper of submillimeter accuracy. The spheres were then embedded into a polystyrene foam block and scanned using a CT scanner (Brilliance CT 64-Channel Scanner, Philips) and/or an MR imaging unit (Discovery 750, GE Healthcare). All of the imaging studies were obtained using a voxel size of $0.5 \times 0.5 \times 1.25 \mathrm{~mm}$ or smaller, according to standard SRS treatment protocols. The geometry and location of the spheres obtained from a scanned image are shown in Fig. 1 upper.

Four randomly selected spheres measuring $0.645 \mathrm{~cm}$, $1.610 \mathrm{~cm}, 2.555 \mathrm{~cm}$, and $3.818 \mathrm{~cm}$ in diameter were initially delineated on the Leksell GammaPlan station for the convenience of data exchange. This system only supported contour export with contour import functionality at the time of the study. The contour points were generated in such a way that the maximum distance along the major axis direction matched the known diameter for a given sphere. These contour data sets were subsequently exported to the other SRS platforms under investigation. Finally, the volumes from the contour data sets were determined on all systems using their respective measurement tools. The measured volumes were compared with each other and also against their physical volumes as determined from known diameters, and further confirmed through a measuring cup filled with water using the Archimedes method.

Similar procedures were carried out for a single clinical case in which the patient had 12 metastatic brain tumors. The 12 tumors were distributed widely throughout the cerebral and cerebellar hemispheres, and both CT and MR imaging had been used in the patient. All tumors were contoured by the attending physician (D.A.L.). Axi- al images showing the distribution of the targets are provided in Fig. 1 lower. The contour data sets for the individual tumors were exported via the DICOM-RT protocol to a stand-alone workstation, as well as to 5 other clinical systems, for volume measurements and comparisons.

To further quantify the results of the aforementioned volume measurements, 10 algorithms were implemented in the stand-alone workstation for independent checks. These algorithms followed 3 general approaches: 1) 3D marching cubes; 2) finite element method; and 3) slice stacking. The key elements of these 3 approaches are described as follows.

\section{Marching Cubes}

For this approach, a grid was first generated to encompass the contour points based on a user-selected coordinate system or from the data itself. Then 3D cubic meshes with fine resolution were generated to cover all the points. Afterward, an isosurface envelope was created to determine whether a voxel fell inside, outside, or was dissected by the enveloping surface. Any dissected voxel volume was decomposed into a set of tetrahedrons using the marching cube algorithm,,$^{13}$ in which the volume of each element was calculated as follows:

$$
V=\frac{1}{6} \sum_{n}|(\vec{a}-\vec{d}) \cdot((\vec{b}-\vec{d}) \times(\vec{c}-\vec{d}))| \quad \text { Eq. } 1
$$

where

$\vec{a}=\left(a_{1}, a_{2}, a_{3}\right), \vec{b}=\left(b_{1}, b_{2}, b_{3}\right), \vec{c}=\left(c_{1}, c_{2}, c_{3}\right), \vec{d}=\left(d_{1}, d_{2}, d_{3}\right)$

are the vectors denoting the 4 vertices of a given tetrahedron, and the summation in Equation 1 was performed over all tetrahedrons intersecting the surface envelope.

\section{Finite Element Method}

For this approach, the triangular isosurface was extracted from the Delauney triangulation of the input contour data. ${ }^{22}$ Then a piecewise linear complex was added to ensure the isosurface would be closed in the shape. A local finite element repairing process was applied to remove isolated vertices, duplicated elements, and nonmanifold vertices. Holes, or subregional labels, were then supplied to make the resulting FEM mesh adaptive to hollow structures. ${ }^{7}$ Two surface mesh sizes, maximum radius of 3 $\mathrm{mm}$ and maximum radius of $1 \mathrm{~mm}$, were tested. Gaussian smoothing was also tested for the method to evaluate its effect on the volume measurement.

\section{Slice Stacking}

Since the input contour data were created in a sliceby-slice manner, slice stacking is a logical step for volume calculation, because this method intrinsically satisfies the initial condition that all input data are distributed in a planar fashion. For this reason, slice stacking can be more straightforward and accurate than a generic 3D approach for the purpose of SRS volume calculation.

When implementing the slice-stacking method, the contour points on each slice were connected either linear- 

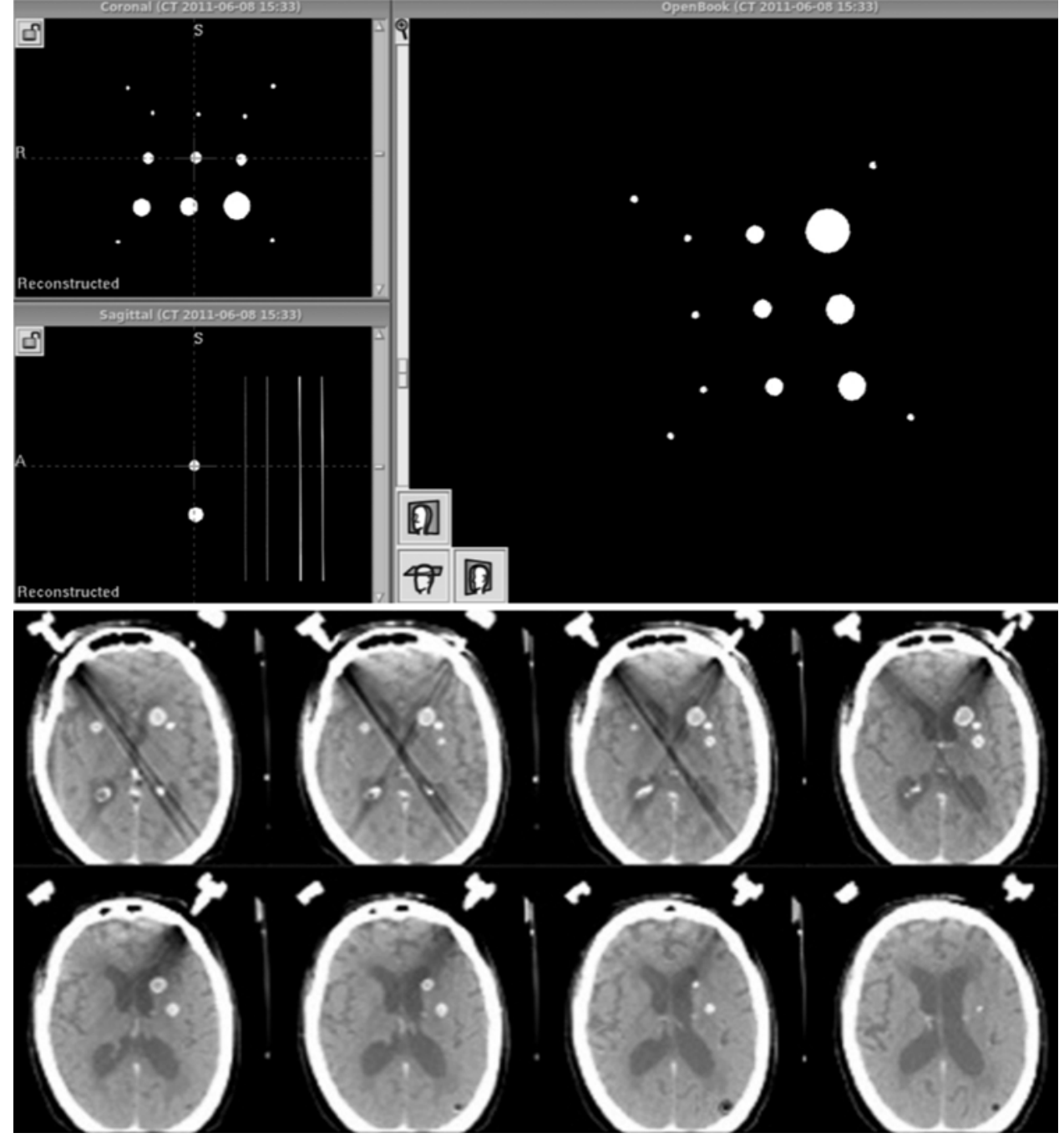

FIG. 1. Computed tomography scans showing a Styrofoam block embedded with acrylic spheres (upper) and the brain of a patient with 12 metastatic lesions (lower).

ly or fitted with a smooth curve such as that interpolated via the B-spline method. ${ }^{6}$ The area of the contour on each slice was then determined using Surveyor's theorem, ${ }^{6}$ and the total volume of the object was calculated as

$V=\frac{1}{2} \sum_{i=0}^{N}\left(S_{i}+S_{i+1}\right) \Delta$

where $N$ is the total number of slices with the contours $\left(\mathrm{S}_{0}\right.$ $=\mathrm{S}_{\mathrm{N}+1}=0$ by definition) and $\Delta$ is the slice thickness. Evidently, Equation 2 approximates the slice volume enclosed by the 2 contours as 2 stepwise n-polygon cylinders with each possessing a height of one-half slice thickness. Note that other partitions in the height of the cylinders, such as one-third or two-thirds of the slice thickness, would yield an identical result.

To improve on the aforementioned stepwise approximation, as imposed on the surface that is mostly continuous and surrounds a structure, we developed the following formula for the slice stacking in addition,
$V=\frac{1}{3} \sum_{i=0}^{N}\left(S_{i}+S_{i+1}+\sqrt{S_{i} S_{i+1}}\right) \Delta$

Eq. 3

where the volume between the $i^{\text {th }}$ and $(i+1)^{\text {th }}$ contours was determined as the volume of a pyramidal/conical frustum using the Cavalieri principle. ${ }^{9}$ Compared with Equation 2, Equation 3 improved on volume handling near the beginning slice and end slice contours. For example, the volume for the end slice extending just beyond the first or the last contour (for example $\mathrm{i}=N$ ) was given as $\mathrm{V}_{\mathrm{N}}=\mathrm{S}_{\mathrm{N}} \Delta / 3$ from Equation 3, matching the volume of a pyramid; whereas Equation 2 yielded a slightly larger volume of $V_{N}=S_{N} \Delta / 2$. Physically, Equation 2 potentially creates a slight overestimation of target volume near the end slices, particularly for the small targets encountered in SRS.

\section{Results}

Figure 2 shows the contoured data plotted in 3D via 

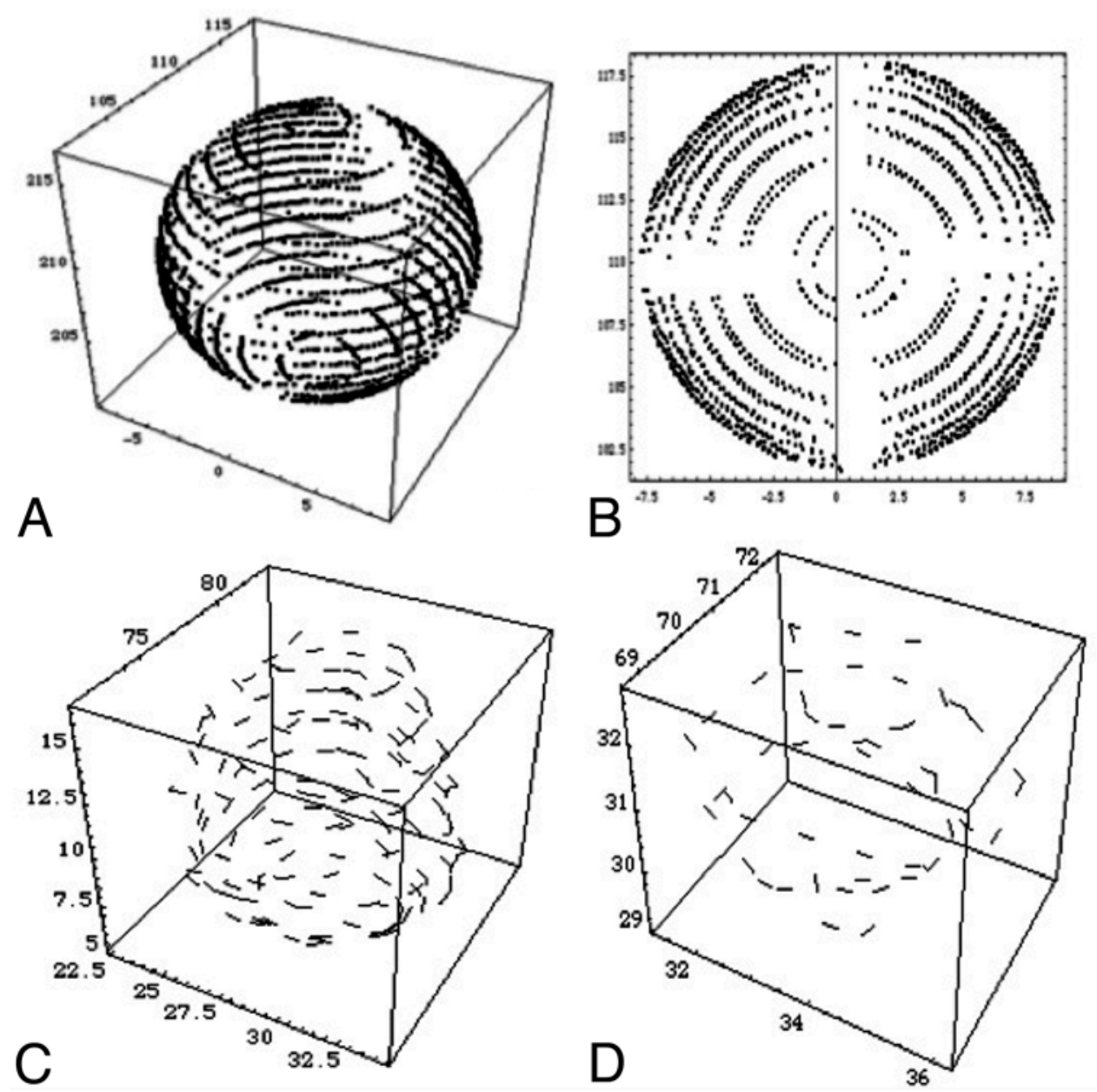

FIG. 2. Plots of contoured data sets for a sphere $(A$ and $B)$ and 2 brain lesions $(C$ and $D)$ from the clinical case reviewed in the study. A: Point-cloud view of a phantom sphere approximately $1.61 \mathrm{~cm}$ in diameter. B: Planar projection of the sphere along the $z$ axis. $\quad$ C: Vector-cloud view of a clinical target approximately $1.09 \mathrm{~cm}$ in diameter. D: Another clinical target approximately $0.40 \mathrm{~cm}$ in diameter. Note the irregularity in the contours near the end slices.

point-cloud distributions. An inspection of the plots and their planar projection (Fig. 2B) found a large irregularity in the end-slice contours even for regular and sizable targets, suggesting a potential pitfall for volume calculation.

The results for the phantom study are shown in Table 1 for cross-platform comparisons, and in Table 2 for cross-algorithm comparisons. The key elements of the algorithms are listed in Table 3. From the results of Tables 1 and 2, consistently higher volumes than the reference values were observed for all platforms including the evaluated algorithms. One clinical platform (No. 4) matched excellently with the reference values, whereas another (No. 2) deviated as much as $20 \%$ from the reference values.

All of the evaluated algorithms in Table 2 exhibited consistently higher volumes than the reference values; however, all of them agreed with each other well. Even for the smallest sphere (Sphere D), all algorithms exhibited a high level of agreement with each other as well as with the reference values. Overall, the hybrid Cavalieri principle-based algorithm (No. 10) and an FEM algorithm (No. 7) produced the closest matches.
The upper panel in Fig. 3 shows results in the clinical case, in which rendered volumes for 12 targets (metastatic lesions) were compared across the 6 clinical SRS platforms. The percentage of variation from the mean value

TABLE 1: Comparison of contour-rendered spherical volumes determined by 6 randomly chosen clinical SRS systems and directly measured reference volumes*

\begin{tabular}{cclll}
\hline \multirow{2}{*}{$\begin{array}{c}\text { Platform } \\
\text { No. }\end{array}$} & \multicolumn{4}{c}{ Volume in $\mathrm{cm}^{3}$ (\% error) $\dagger$} \\
\cline { 2 - 5 } & Sphere A & \multicolumn{1}{c}{ Sphere B } & \multicolumn{1}{c}{ Sphere C } & \multicolumn{1}{c}{ Sphere D } \\
\hline 1 & $29.80(2.3)$ & $9.01(1.7)$ & $2.20(0.0)$ & $0.142(1.4)$ \\
2 & $30.65(5.2)$ & $9.38(5.9)$ & $2.41(9.6)$ & $0.171(22.1)$ \\
3 & $29.82(2.4)$ & $9.01(1.7)$ & $2.25(2.3)$ & $0.142(1.4)$ \\
4 & $29.23(0.3)$ & $8.79(-0.8)$ & $2.19(-0.45)$ & $0.141(0.7)$ \\
5 & $30.04(3.1)$ & $9.13(3.1)$ & $2.30(4.6)$ & $0.154(10.0)$ \\
6 & $29.84(2.4)$ & $9.03(1.9)$ & $2.26(2.7)$ & $0.150(7.1)$ \\
ref value & 29.13 & 8.86 & 2.20 & 0.140 \\
\hline
\end{tabular}

${ }^{*}$ ref $=$ reference.

$\dagger$ Percentage errors compared with reference values. 
TABLE 2: Comparison of sphere volume calculations made using 10 algorithms and directly measured reference volumes

\begin{tabular}{ccccc}
\hline \multirow{2}{*}{$\begin{array}{c}\text { Algorithm } \\
\text { No. }\end{array}$} & \multicolumn{4}{c}{ Volume in $\mathrm{cm}^{3}(\%$ error) } \\
\cline { 2 - 5 } & Sphere A & Sphere B & Sphere C & Sphere D \\
\hline 1 & $29.87(2.5)$ & $9.01(1.7)$ & $2.27(3.2)$ & $0.146(4.3)$ \\
2 & $29.89(2.6)$ & $9.04(2.0)$ & $2.27(3.2)$ & $0.147(5.0)$ \\
3 & $29.85(2.5)$ & $9.03(1.9)$ & $2.27(3.2)$ & $0.145(3.6)$ \\
4 & $29.87(2.5)$ & $9.04(2.0)$ & $2.26(2.7)$ & $0.145(3.6)$ \\
5 & $29.80(2.3)$ & $9.00(1.6)$ & $2.25(2.3)$ & $0.135(-3.6)$ \\
6 & $29.86(2.5)$ & $9.02(1.8)$ & $2.25(2.3)$ & $0.144(2.9)$ \\
7 & $29.80(2.3)$ & $8.99(1.5)$ & $2.25(2.3)$ & $0.135(-3.6)$ \\
8 & $29.80(2.3)$ & $9.02(1.8)$ & $2.25(2.3)$ & $0.143(2.1)$ \\
9 & $29.86(2.5)$ & $9.02(1.8)$ & $2.25(2.3)$ & $0.143(2.1)$ \\
10 & $29.83(2.4)$ & $9.00(1.6)$ & $2.24(1.8)$ & $0.141(0.7)$ \\
ref value & 29.13 & 8.86 & 2.20 & 0.140 \\
\hline
\end{tabular}

* Percentage errors compared with reference values.

of each target is also given in Fig. 3 lower. A power law fit to the data yielded $\mathrm{R}^{2}=0.904$ in the semilogarithmic scale and provided a general trend of the data. Overall, an approximately 3\%-10\% variation in the rendered target volume was noted, with smaller volumes yielding larger variations.

The results shown in Fig. 3 were further confirmed by those in Fig. 4, in which the same targets are compared across 10 volume-calculation algorithms implemented from 3 general types of methods described in the Methods. Variations in the algorithms are categorized in the figure caption. Similar trends of the data were noted for Fig. 4, compared with Fig. 3. The power fit of the data yielded $\mathrm{R}^{2}=0.939$. The percentage of variation was approximately $1 \%-6 \%$, and it increased with decreasing target volumes. This decrease gradually leveled, and the turning point for targets shown in both Figs. 3 and 4 was determined at a volume of approximately $0.4 \mathrm{~cm}^{3}$. Note
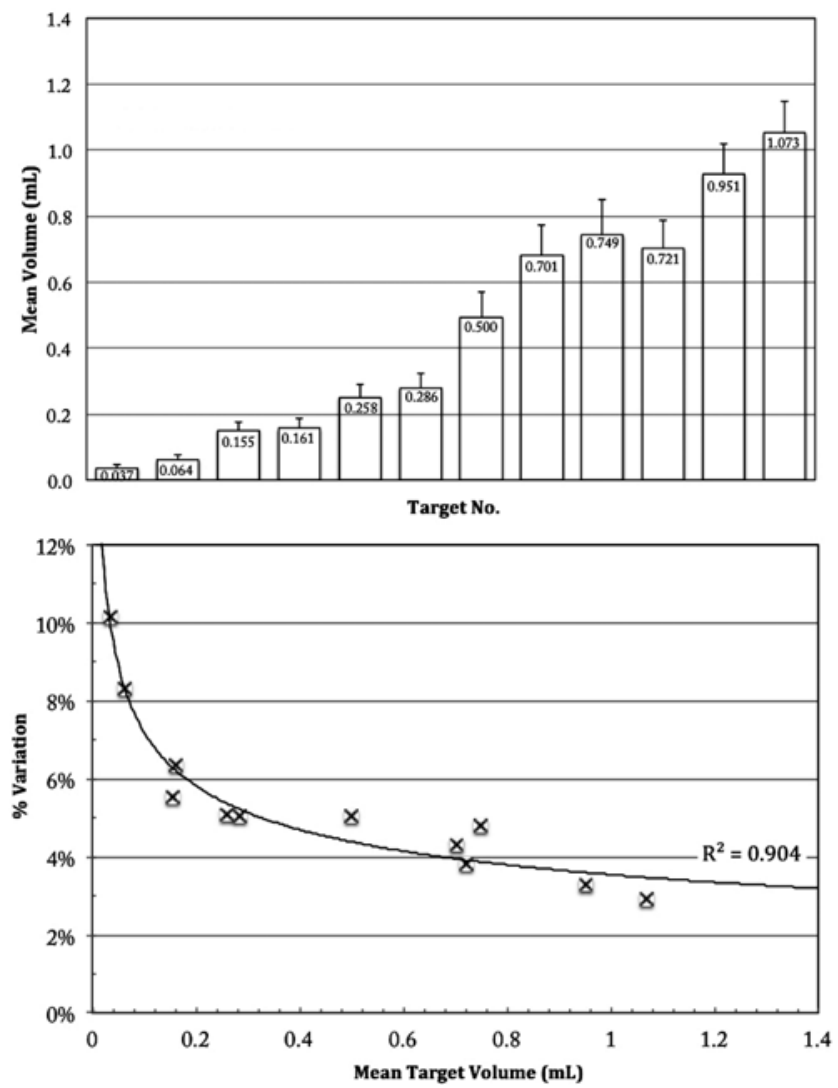

FIG. 3. Clinical platform comparisons. Variations in target volumes among the SRS platforms used in the clinical case. Upper: Bar graph with the mean values indicated for individual targets. Lower: Percentage variation from the mean value for all targets. The solid line is the power fit of the data with the correlation $\left(R^{2}\right)$ value shown.

that the mean target volumes listed on the bar plots in Fig. 4 were in excellent agreement with those in Fig. 3, which highlights the consistency of our results.

To highlight the importance of end-slice handling,

TABLE 3: Comparison of key features of implemented volume computation algorithms*

\begin{tabular}{|c|c|c|c|}
\hline $\begin{array}{l}\text { Algorithm } \\
\text { No. }\end{array}$ & $\begin{array}{c}\text { Contour Points } \\
\text { Handling }\end{array}$ & Implementation Method & Key Elements \\
\hline 1 & 2D & trapezoidal approximation & slice area computed via convex hull approximation \\
\hline 2 & $2 \mathrm{D}$ & B-spline curve fitting & slice contour points connected via 2D curve fitting \\
\hline 3 & $3 \mathrm{D}$ & rigid cubic lattice & direct voxel counting; voxel size = image resolution \\
\hline 4 & 3D & rigid cubic lattice & direct voxel counting; voxel size = image resolution $/ 3$ \\
\hline 5 & 3D & marching cube & tetrahedron surface meshing; voxel size = image resolution \\
\hline 6 & 3D & marching cube & tetrahedron surface meshing; voxel size = image resolution $/ 3$ \\
\hline 7 & $3 \mathrm{D}$ & FEM & local surface meshing via $R_{\max }=3 \mathrm{~mm}$ \\
\hline 8 & $3 \mathrm{D}$ & FEM & local surface meshing via $R_{\max }=1 \mathrm{~mm}$ \\
\hline 9 & $3 \mathrm{D}$ & FEM & $\begin{array}{l}\text { local surface meshing via } R_{\max }=1 \mathrm{~mm}+\text { gaussian smoothing }(\sigma=3 \times \\
\text { average vertex-to-edge distance) }\end{array}$ \\
\hline 10 & hybrid & $\begin{array}{l}\text { frustum surface approxi- } \\
\text { mation }\end{array}$ & slice contours connected via frustum surface approximation \\
\hline
\end{tabular}

${ }^{*} R_{\max }=$ Maximum radius of the local surface mesh. 

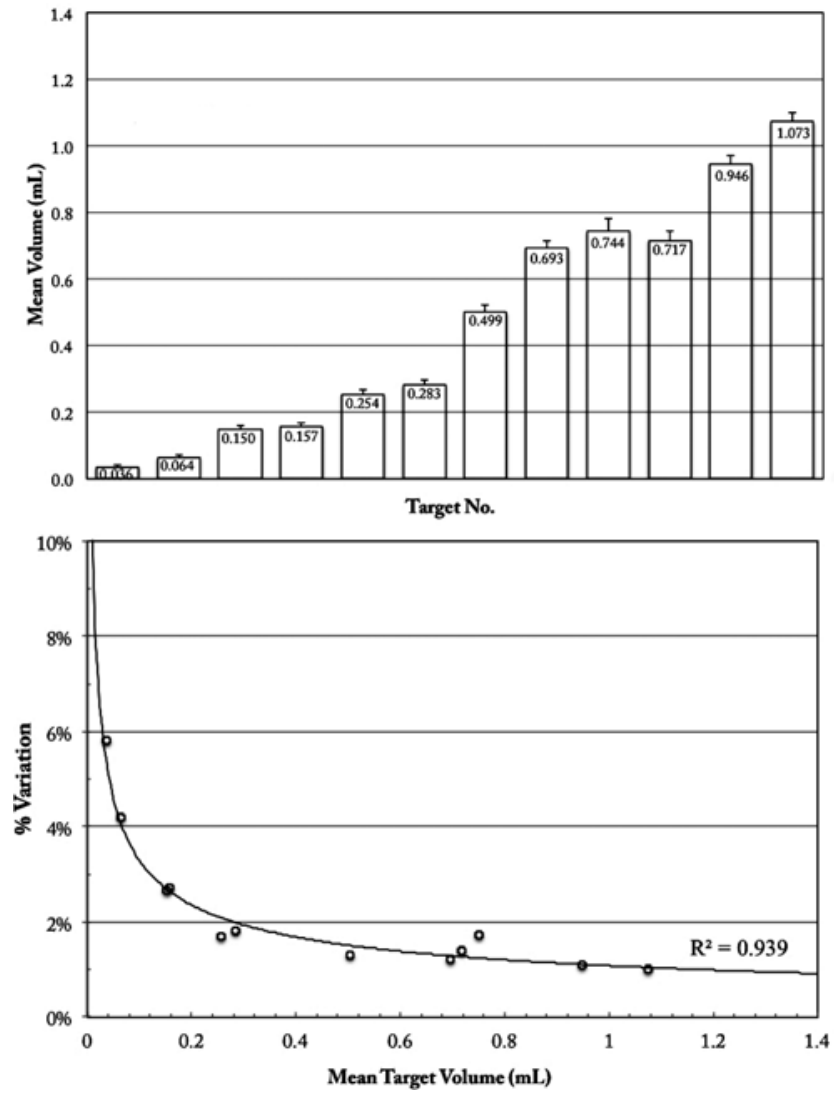

FIG. 4. Algorithm comparisons. Results of rendered target volumes from 10 different algorithms used in the clinical case. Upper: Bar graph demonstrating variations in volumes of the targets. Lower: Percentage variation from the mean value obtained using a power formula fitting similar to that shown in Fig. 3.

Fig. 5 shows a graph plotting the serial area function versus the slice number for 2 targets, as determined from the raw data of Equation 2 (solid line) and the fitted data of Equation 3 (dashed line). The line projection plots of the contour data illustrated the size and shape of the 2 targets. Note that little difference was noted between the 2 algorithms for the relatively large target (Fig. 5 upper), whereas there was a significant difference for the relatively small target (Fig. 5 lower). Such an observation also supported the results of Figs. 3 and 4.

\section{Discussion}

Our study has demonstrated large contour-based volume calculation variability among current SRS systems. To understand such device-dependent results, we tested a variety of volume calculation algorithms, which included different approaches, for example, slice-by-slice stacking, linear interpolation, smooth curve fitting, varying voxel size, cubic and tetrahedron meshing, and 3D point cloud with or without surface smoothing.

We conclude that there are several potential pitfalls in volume calculation based on the methods used in this study. First, proper end-slice handling is important for SRS targets and, second, a proper grid size (or kernel size) is important for 3D-based volume calculation (such as the marching-cube algorithm) to avoid oversimplifica-
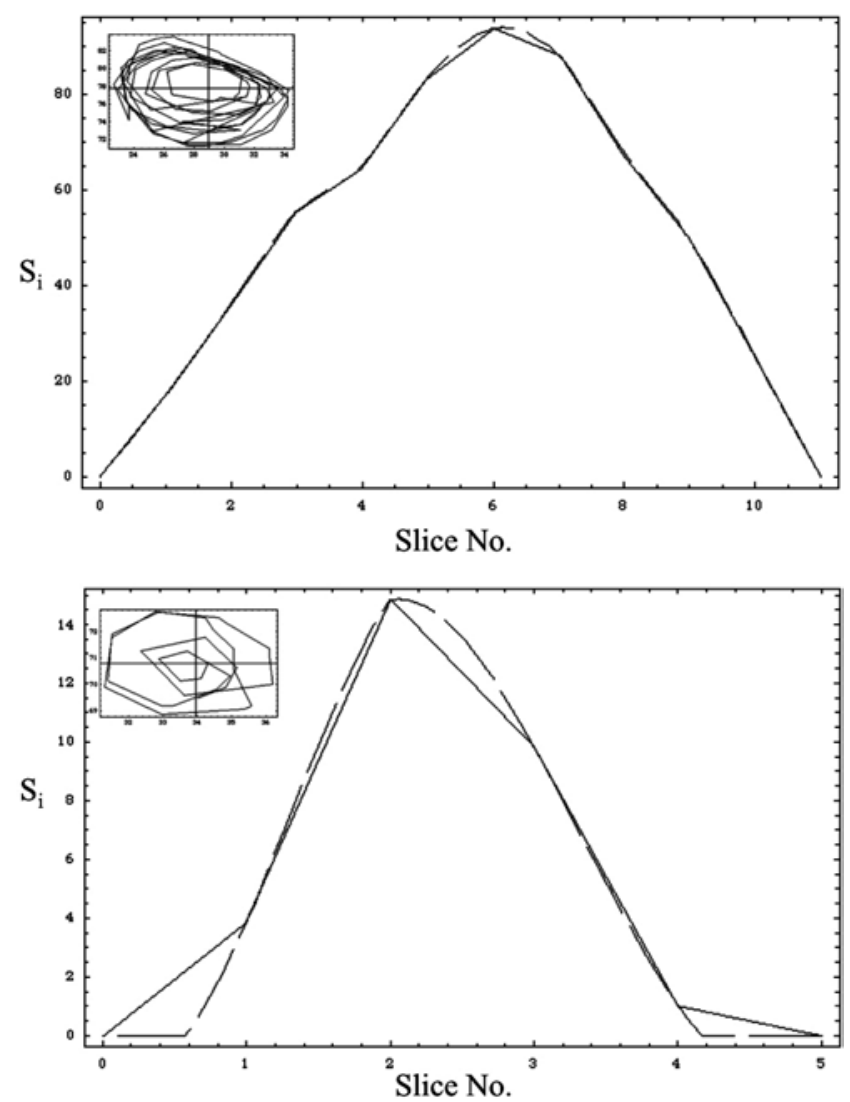

FIG. 5. Illustration showing implementation of a Cavalieri principlebased algorithm in handling the end-slice contours. Upper: Serial slice area function for the target illustrated in Fig. 2C. Lower: Serial slice area function for the target illustrated in Fig. 2D. The planar contour projection plots are shown in the insets. Note that the target volume equals the area integrated under the curve, whereas the solid line shows the non-smoothed surface result and the dashed line shows the smoothed surface result.

tion of a shape possessing sharp edges or large changes between slices. In our study, such a grid or kernel size was governed by the spacing between adjacent contour points, and any downsampling was not found to improve the results significantly.

Despite these pitfalls, all algorithms performed consistently (within 3\%) with each other except when dealing with small targets of $0.4 \mathrm{~cm}^{3}$ or less, in which case the discrepancy approximately doubled. We conclude that the overall variability among mathematical volume-calculation algorithms tracked well with the level of discrepancy observed across clinical SRS platforms. However, whether the clinical SRS systems under investigation actually adopted a calculation or an empirical model-based correction for volume calculation is unknown to us. Furthermore, other sources of uncertainty, such as contour delineation under different window and level settings, were not investigated. For example, spherical contours were taken to represent the shape of each object only on the basis of independent diameter measurements. For the purposes of the study, the volume of an object was also assumed to be solely dependent on the contour points, regardless of the SRS system being investigated. 


\section{Volume calculation for intracranial stereotactic radiosurgery}

One potential source of uncertainty lies in the contour points resampling error, which potentially occurs when contour points from one system are imported into another. This phenomenon was investigated using Algorithm No. 2, in which all data were resampled via the Bspline fitting. No significant variation in volume calculations was found, which confirmed sufficient data points delineated for all objects in the current study. Nonetheless, a resampling error from a limited number of slices could significantly affect the volume calculations for small objects that appear only on a few slices. The results shown in Figs. 3 and 4 supported such a finding, despite the fact that more complex algorithms were used in our study than simple 2D trapezoidal rule-based algorithms such as Algorithm No. 1 and those reported in a previous study. ${ }^{24}$

From the results of this study, it was evident that the existing contour-based planar data structure, which originated in the DICOM-RT protocol, is not sufficient to guarantee consistent and accurate volume calculation across SRS platforms. Additional information is needed, as are specifications such as embedding key slice-stacking parameters for volume calculation in the protocol to ensure consistent results across platforms. Until any standard becomes available, it is imperative for users to perform careful quality assurance and benchmarking studies of an SRS system with respect to volume calculation accuracy, before its initial use for clinical practice as well as for conducting cross-platform studies.

Although our study has focused on volume calculation from user-defined target/object contour data sets, the general problems and pitfalls remain for volume calculation of $3 \mathrm{D}$ distributions, including isodose volumes or surfaces. It is known that bin size, or voxel size, affects the results of dose-volume histogram calculations and reference dose calculations..$^{14}$ As SRS evolves to treat multiple brain lesions - 10 or more-any inaccuracy or inconsistency in volumes of individual targets would compound the uncertainty concerning total volume of targets and surrounding normal structures. This may significantly affect the tolerance of normal tissue as well as the dose prescribed for the treatment. ${ }^{20}$

\section{Conclusions}

We found significant variability among the SRS systems we studied in their contour-based volume calculation results. This suggests the need for a uniform standard or consensus in data-transferring protocols to eliminate such systematic uncertainties.

\section{Disclosure}

Dr. McDermott shares a copyright with the University of California, San Francisco, for a medical device. Dr. Larson is a stockholder and member of the board of directors for American Shared Hospital Services. No other author reports any potential conflict of interest.

Author contributions to the study and manuscript preparation include the following. Conception and design: Ma, Sahgal, Shrieve, Sneed, McDermott, Larson. Acquisition of data: Ma, Sahgal, Nie,
Hwang, Karotki, Wang, Shrieve, Sneed, Larson. Analysis and interpretation of data: Ma, Sahgal, Nie, Hwang, Karotki, Wang, Shrieve, McDermott, Larson. Drafting the article: Ma, Sahgal, Nie, Larson. Critically revising the article: all authors. Reviewed submitted version of manuscript: all authors. Approved the final version of the manuscript on behalf of all authors: Ma. Administrative/technical/ material support: Ma, Shrieve, Sneed, McDermott, Larson. Study supervision: Ma, McDermott, Larson.

\section{References}

1. Adler JR Jr, Chang SD, Murphy MJ, Doty J, Geis P, Hancock SL: The Cyberknife: a frameless robotic system for radiosurgery. Stereotact Funct Neurosurg 69:124-128, 1997

2. Andrews DW, Bednarz G, Evans JJ, Downes B: A review of 3 current radiosurgery systems. Surg Neurol 66:559-564, 2006

3. Andrews DW, Scott CB, Sperduto PW, Flanders AE, Gaspar LE, Schell MC, et al: Whole brain radiation therapy with or without stereotactic radiosurgery boost for patients with one to three brain metastases: phase III results of the RTOG 9508 randomised trial. Lancet 363:1665-1672, 2004

4. Bedford JL, Childs PJ, Nordmark Hansen V, Mosleh-Shirazi MA, Verhaegen F, Warrington AP: Commissioning and quality assurance of the Pinnacle(3) radiotherapy treatment planning system for external beam photons. Br J Radiol 76:163176, 2003

5. Bharat S, Parikh P, Noel C, Meltsner M, Bzdusek K, Kaus M: Motion-compensated estimation of delivered dose during external beam radiation therapy: implementation in Philips' Pinnacle(3) treatment planning system. Med Phys 39:437443, 2012

6. Braden B: The Surveyor's area formula. Coll Math J 17:326337, 1986

7. Fang Q, Boas D: Tetrahedral mesh generation from volumetric binary and grayscale images, in: Proceedings of the 6th IEEE International Symposium on Biomedical Imaging: From Nano to Marco, June 28-July 1, 2009. Boston: ISBI, 2009, pp 1142-1145

8. Follwell MJ, Khu KJ, Cheng L, Xu W, Mikulis DJ, Millar BA, et al: Volume specific response criteria for brain metastases following salvage stereotactic radiosurgery and associated predictors of response. Acta Oncol 51:629-635, 2012

9. Harris J, Stocker H: Handbook of Mathematics and Computational Science. New York: Springer-Verlag, 1998

10. Jin JY, Yin FF, Ryu S, Ajlouni M, Kim JH: Dosimetric study using different leaf-width MLCs for treatment planning of dynamic conformal arcs and intensity-modulated radiosurgery. Med Phys 32:405-411, 2005

11. Leksell L: The stereotaxic method and radiosurgery of the brain. Acta Chir Scand 102:316-319, 1951

12. Lindquist C, Paddick I: The Leksell Gamma Knife Perfexion and comparisons with its predecessors. Neurosurgery 61 (3 Suppl):130-141, 2007

13. Lorensen W, Cline H: Marching Cubes: a high resolution 3D surface reconstruction algorithm. Comput Graph 21:163169,1987

14. Ma L, Chin LS, Shepard D, Amin P, Slawson R: The effect of user-defined variables on dosimetry consistency in Gamma Knife planning. Phys Med Biol 45:N43-N47, 2000

15. Ma L, Petti P, Wang B, Descovich M, Chuang C, Barani IJ, et al: Apparatus dependence of normal brain tissue dose in stereotactic radiosurgery for multiple brain metastases. Technical note. J Neurosurg 114:1580-1584, 2011

16. Mackie TR, Balog J, Ruchala K, Shepard D, Aldridge S, Fitchard E, et al: Tomotherapy. Semin Radiat Oncol 9:108117, 1999

17. Mackie TR, Holmes T, Swerdloff S, Reckwerdt P, Deasy JO, Yang J, et al: Tomotherapy: a new concept for the delivery of dynamic conformal radiotherapy. Med Phys 20:1709-1719, 1993 
18. Paddick I: A simple scoring ratio to index the conformity of radiosurgical treatment plans. Technical note. J Neurosurg 93 (Suppl 3):219-222, 2000

19. Paddick I, Lippitz B: A simple dose gradient measurement tool to complement the conformity index. J Neurosurg 105 Suppl:194-201, 2006

20. Sahgal A, Barani IJ, Novotny J Jr, Zhang B, Petti P, Larson DA, et al: Prescription dose guideline based on physical criterion for multiple metastatic brain tumors treated with stereotactic radiosurgery. Int J Radiat Oncol Biol Phys 78:605608, 2010

21. Salter BJ, Fuss M, Sarkar V, Wang B, Rassiah-Szegedi P, Papanikolaou N, et al: Optimization of isocenter location for intensity modulated stereotactic treatment of small intracranial targets. Int J Radiat Oncol Biol Phys 73:546-555, 2009

22. Seidel R: The upper bound theorem for polytopes: an easy proof of its asymptotic version. Comput Geom 5:115-116, 2009

23. Shaw E, Scott C, Souhami L, Dinapoli R, Kline R, Loeffler J, et al: Single dose radiosurgical treatment of recurrent previously irradiated primary brain tumors and brain metastases: final report of RTOG protocol 90-05. Int J Radiat Oncol Biol Phys 47:291-298, 2000

24. Snell JW, Sheehan J, Stroila M, Steiner L: Assessment of imaging studies used with radiosurgery: a volumetric algorithm and an estimation of its error. Technical note. J Neurosurg 104:157-162, 2006
25. Therasse P, Arbuck SG, Eisenhauer EA, Wanders J, Kaplan RS, Rubinstein L, et al: New guidelines to evaluate the response to treatment in solid tumors. European Organization for Research and Treatment of Cancer, National Cancer Institute of the United States, National Cancer Institute of Canada. J Natl Cancer Inst 92:205-216, 2000

26. Werner-Wasik M, Nelson AD, Choi W, Arai Y, Faulhaber PF, Kang P, et al: What is the best way to contour lung tumors on PET scans? Multiobserver validation of a gradient-based method using a NSCLC digital PET phantom. Int J Radiat Oncol Biol Phys 82:1164-1171, 2012

Manuscript submitted May 16, 2012.

Accepted July 10, 2012.

This work was presented in part in abstract form at the 16th International Meeting of the Leksell Gamma Knife Society, March 25-29, 2012, in Sydney, Australia.

Please include this information when citing this paper: DOI: 10.3171/2012.7.GKS121016.

Address correspondence to: Lijun Ma, Ph.D., Department of Radiation Oncology, University of California, San Francisco, 505 Parnassus Avenue, Room L-08, San Francisco, California 94143. email: lijunma@radonc.ucsf.edu. 